Instructions for authors, subscriptions and further details:

http://rasp.hipatiapress.com

\title{
Situación Familiar, Protección Social y Bienestar de las Personas Mayores en Perspectiva Europea Comparada
}

Almudena Moreno Mínguez ${ }^{1}$, Juan Antonio Vicente Virseda ${ }^{2}$

1) Universidad de Valladolid, España

2) Universidad Nacional de Educación a Distancia, España

Date of publication: July $30^{\text {th }}, 2016$

Edition period: July 2016 - January 2017

To cite this article: Moreno, A. \& Vicente, J. A. (2016). Situación Familiar, Protección Social y Bienestar de las Personas Mayores en Perspectiva Europea Comparada. Research on Ageing and Social Policy, 4(2), 96-127. doi: 10.17583/rasp.2016.2048

To link this article: http://doi.org/10.17583/rasp.2016.2048

\section{PLEASE SCROLL DOWN FOR ARTICLE}

The terms and conditions of use are related to the Open Journal System and to Creative Commons Attribution License (CCAL). 


\section{Situación Familiar, Protección Social y Bienestar de las Personas Mayores en Perspectiva Europea Comparada}
Almudena Moreno Mínguez
Juan Antonio Vicente Virseda
Universidad de Valladolid
Universidad Nacional de Educación a Distancia

\section{Resumen}

El objetivo de esta investigación es analizar el bienestar y la calidad de vida de las personas mayores de más de 65 que viven en hogares multigeneracionales en comparación con las que viven en su propio hogar en pareja o solos, así como las diferencias existentes entre países respecto al bienestar subjetivo de estas personas, teniendo como telón de fondo el marco del Estado de bienestar, las políticas sociales de dependencia y los modelos familiares en perspectiva comparada. La metodología empleada se basa en análisis descriptivos y análisis de asociación utilizando para ello la denominada técnica "árboles de decisión" a partir de los datos procedentes de la encuesta European Quality of Life, 2011-2012. Los hallazgos apuntan a que vivir en hogares multigeneracionales está inversamente relacionado con la percepción del bienestar y la calidad de vida en el colectivo de personas mayores de 65 años en los países del sur de Europa. De hecho, los resultados sugieren que las políticas sociales de los Estados de bienestar en el norte de Europa contribuyen al bienestar de estas personas en mayor medida que la solidaridad familiar y la corresidencia con los familiares, características propias de los países mediterráneos.

Palabras clave: hogares multigeneracionales, bienestar, política social, Europa, personas mayores. 


\section{Family Situation, Social Protection, and Well-being of Older Adults from an European Comparative Perspective}

\author{
Almudena Moreno Mínguez \\ Universidad de Valladolid
}

Juan Antonio Vicente Virseda

Universidad Nacional de Educación a Distancia

\section{Abstract}

The aim of this research is therefore to analyse the well-being of people over 65 living in multi-generational households, compared to those who live in their own home, either with a partner or alone. The analysis takes a comparative approach, and seeks to analyse and unravel the differences between countries in terms of the subjective well-being of this segment according to the type of household they live in, against a backdrop of the framework of the welfare state, social policies on dependent care, and family models. The methodology used in this comparative approach consists of applying association analyses based on "decision trees". The data source used is the European Quality of Life Survey, 2011-2012. The findings show that live in multigenerational households is inversely related to the perception of well-being and quality of life in the group of people over 65 years in the countries of southern Europe. Indeed the results suggest that the social policies implemented by welfare States can satisfactorily contribute to the well-being and quality of life of these groups in the same or greater measure as family solidarity, as evidenced by the results obtained for Nordic countries.

Keywords: multigenerational household, well-being, social policy, Europe, ageing. 
$\mathrm{E}$ 1 primer trabajo empírico sobre las tipologías familiares en Europa es el trabajo histórico de Laslett (1972), quien por primera vez se refiere a los hogares multigeneracionales o familias extensas. De acuerdo con Hammel y Laslett (1974), la familia extensa es conceptualizada por Naciones Unidas, 2007, como un hogar que tiene más de un núcleo familiar, también denominada "multiple family household". Este tipo de núcleos familiares son característicos en los países del sur de Europa y Reino Unido, país este último donde se contabilizaba al servicio doméstico como parte de la familia.

La familia extensa ha sido objeto de estudio reciente como prototipo característico de los países del sur de Europa, caracterizados por una reducida protección del Estado del bienestar a las familias (Ferrera, 1996; Leibfried, 1992; Moreno y Serrano, 2009), la limitada secularización y la permanencia de valores tradicionales (Inglehart, 2003), factores que han contribuido a fortalecer los lazos familiares y la solidaridad intergeneracional (Reher, 1998, Moreno Mínguez, 2013; Calzada, 2013).

En este contexto de análisis nos encontramos con numerosos trabajos de investigación que han destacado la existencia de un modelo de familia mediterránea caracterizado, entre otros factores, por la solidaridad intergeneracional y por la moderada incidencia de los hogares multigeneracionales en comparación con otros países europeos (Carrascosa y Sancho, 2013; Naldini, 2003, Iacovou y Skew, 2011; Eurofound, 2014; Zueras y Miret, 2013). Si bien los estudios sugieren que el fenómeno de los hogares multigeneracionales es más frecuente en los países del sur de Europa que en el norte de Europa (Koslowski, 2009; Tomassini et al., 2004), son más bien escasos los estudios disponibles que analicen la relación entre las prácticas de solidaridad intergeneracional en las familias extensas y el bienestar de las personas que residen en este tipo de hogares (Albuquerque, 2011; Hank y Buber, 2009; Koslowski, 2009).

En este proceso de cambio familiar, los hogares multigeneracionales han experimentado un descenso en la mayoría de los países europeos, pero no así la frecuencia de relaciones entre generaciones que ha cobrado relativa intensidad (Bengtson, 2001; Hagestad, 2006). En concreto, Bengston (2001) se refiere a seis dimensiones de la solidaridad intergeneracional dentro de las familias (afectuosa, consensuada, normativa, funcional, estructural y asociativa). En el caso de los hogares multigeneracionales, Alburqueque 
(2008) destaca que todas estas dimensiones de las relaciones intergeneracionales incrementan el bienestar de los individuos y de la sociedad en general.

En base a esta interpretación, cabría esperar que, en aquellos países donde los hogares multigeneracionales son más frecuentes y donde mayor porcentaje de personas mayores de sesenta y cinco años viven en este tipo de hogares, mayor será el nivel de bienestar de estas personas en comparación con otros países donde las familias multigeneracionales y la solidaridad intergeneracional está menos arraigada.

Son numerosos los estudios que han abordado el bienestar subjetivo de este grupo de edad desde diferentes perspectivas utilizando diversos indicadores (Khan y Raeside, 2014; Stone et al, 2010; Deaton, 2008; Ulloa, 2014; Wikman et al., 2011). De acuerdo con estos estudios, el bienestar subjetivo y el estado de salud son dos factores estrechamente relacionados en las personas mayores de 65 años (Bowling, 1997; Smith et al., 2002; Yang et al., 2012; Connell et al., 2012). Según Hafiz et al. (2014), la edad, el sexo y el estado de salud son predictores de la calidad de vida en estas personas. Sin embargo, según numerosas investigaciones, la calidad de vida es una medida del bienestar general de la vida humana que responde a un concepto multidimensional que incluye, además de la salud, las relaciones sociales y las condiciones materiales de vida, las percepciones y valoraciones subjetivas (CDC, 2013; Moreno Mínguez et al., 2014; OCDE 2010a; 2010b; 2011; Dolan et al., 2008).

La incidencia de estos factores en la calidad de vida y bienestar de este grupo de edad ha sido ampliamente estudiada en la literatura (Green et al., 1992; Singh y Misra, 2009). Sin embargo, conocemos muy poco en términos científicos sobre la relación existente entre las formas de corresidencia, la dependencia familiar y la calidad de vida y bienestar de estas personas en perspectiva comparada. En este artículo nos vamos a ocupar precisamente de analizar cómo influye el tipo de hogar en el que viven estas personas en el bienestar percibido o también denominado "bienestar subjetivo" y, por tanto, en su calidad de vida.

Las investigaciones realizadas a tal efecto destacan que las tendencias observadas sobre el bienestar percibido no son homogéneas entre los diferentes países porque intervienen numerosos factores culturales, institucionales, económicos y familiares (Otero et al., 2004). Entre estos factores destacan el papel de la red de solidaridad familiar y la naturaleza de 
los regímenes de cuidado (Bettio, 2004; Carrascosa, 2011; Viazzo, 2003). En cuanto a las redes familiares, los datos muestran que los países del sur de Europa se caracterizan por tener una red familiar de apoyo a la tercera edad, prácticamente inexistente en los países del norte de Europa (Saraceno, 2008; Daatland y Herlofson, 2003). Por el contrario, en los países del norte de Europa la red de voluntariado de ayuda a la tercera edad es más profesional y frecuente que en los países mediterráneos (Erlinghagen y Hank, 2006).

Aunque contamos con relativamente pocos estudios que analicen el bienestar de las personas mayores de 65 años en relación con el tipo de hogar en el que viven, algunas investigaciones han aportado conclusiones de interés. Los análisis de Aranda (2013) han evidenciado que vivir en un hogar multigeneracional reduce el estado depresivo de estas personas en los países del sur de Europa, donde los lazos familiares de solidaridad son muy fuertes. Sin embargo, para los países del norte de Europa no se detecta tal asociación significativa entre las formas residenciales de los ancianos y su estado de ánimo depresivo.

Por otro lado, contamos con otros estudios que concluyen que, en los países mediterráneos, donde los lazos familiares son muy intensos y las familias extensas son más numerosas que en otros países europeos, el sentimiento de soledad es mayor. Por ejemplo, estos estudios han revelado que los italianos mayores de sesenta y cinco años se sienten más solos que los holandeses, a pesar de que estos últimos viven solos en un porcentaje elevado (Van Tilburg, Gierveld, Lecchini, y Marsella, 1998).

Esto podría explicarse por las elevadas expectativas que tienen de recibir apoyo familiar y la correspondiente mayor sensación de decepción cuando estas expectativas no se cumplen en los países del sur de Europa (Litwin, 2010; Kimm et al., 2014). Van Tilburg et al. (1998) consideran que los sentimientos de soledad tienden a ser más frecuentes en las sociedades comunales en las que hay mayores expectativas para el apoyo familiar.

En base a esta revisión teórica, el principal objetivo de este estudio es explorar la posible asociación entre el tipo de hogar en que viven las personas mayores de 65 años y el bienestar percibido en el marco comparado de la política social desarrollada por los diferentes Estados de bienestar y en base a una agrupación previa de los países en función del tipo de hogar predominante en el que viven estas personas. Para conseguir este fin, en primer lugar, se presenta un análisis descriptivo comparado sobre las 
tipologías de hogares, la protección social destinada a este grupo de edad y el índice de bienestar a través de la revisión de los indicadores existentes.

En una segunda fase analítica, se explora la posible correlación entre vivir solo, en pareja o en un hogar multigeneracional en relación con los diferentes indicadores definidos de bienestar y calidad de vida en perspectiva comparada.

\section{Metodología y Fuentes de Datos}

La estrategia analítica utilizada se ha basado básicamente en combinar dos instrumentos metodológicos de distinta naturaleza. Por un lado, se ha realizado un análisis descriptivo comparado sobre las formas residenciales de las personas mayores de 65 años, el grado de protección social y el bienestar subjetivo. Por otro lado, se ha realizado un análisis exploratorio más avanzado a partir de análisis de asociación, en el que se han introducido variables relativas a las formas residenciales y las variables relacionadas con el bienestar subjetivo a partir de los datos contenidos en la European Quality of Life Survey del año 2012 (EQLS).

El análisis descriptivo se ha realizado comparando varios indicadores relativos a la morfología familiar (tipo de hogar en el que viven las personas mayores de 65 años) y la protección social destinada a este grupo de edad (gastos sociales en tercera edad y en dependencia). Estos datos proceden de diferentes fuentes como la European Union Statistics on Income and Living Conditions (EU-SILC) de Eurostat y de la OECD Health Statistics. Finalmente, se utilizará el índice de bienestar elaborado por Kaneda, Lee y Pollard (2011) para los países recogidos en dicho informe. Este índice sintético se basa en indicadores de bienestar material, físico, social y emocional. Los datos para construir este índice provienen de la Encuesta realizada entre 2004 y 2006 en Europa, Survey of Health, Ageing and Retirement (SHARE).

El análisis presentado en la segunda parte se basa en los datos procedentes de los países participantes en la tercera oleada de la European Quality of Life Survey (EQLS) en 2012. Esta encuesta se realizó en 27 países de la Unión Europea sobre una muestra de 43.636 entrevistados. El cuestionario recoge diferentes dimensiones de la calidad de vida y el bienestar, centrándose en aspectos como las relaciones personales y sociales, 


\section{Moreno \& Vicente - Situación Familiar de las Personas Mayores}

el grado de satisfacción, el estado de salud percibido y el estado de ánimo, entre otras variables definidas en el modelo. ${ }^{1}$ A partir del cuestionario y de los objetivos propuestos, hemos definido los siguientes indicadores:

1) Ocio y calidad de las relaciones sociales.

2) Percepción de bienestar subjetivo (satisfacción percibida de la vida).

3) Calidad de vida (estado de salud y autonomía física).

4) El bienestar emocional (estado de ánimo).

En base a estos indicadores hemos seleccionado cuatro variables relacionadas con la calidad de las relaciones sociales y de ocio: percepción del tiempo disfrutado con la familia, relaciones con los amigos y vecinos, aficiones y trabajo voluntario.

La percepción del bienestar subjetivo se ha computado a través de la satisfacción del entrevistado con varias dimensiones, como el nivel de vida actual, el alojamiento, la familia, la salud, la situación económica en el país y la felicidad percibida.

Hemos utilizado tres variables relacionadas con la calidad de vida: la percepción del estado de salud, la tenencia de enfermedades crónicas o problemas de salud y las actividades diarias que se ven limitadas por problemas de salud.

Por último, hemos utilizado algunas variables relacionadas con el bienestar emocional: sentimiento de alegría, de sentirse relajado, activo y vigoroso, descansado, entusiasta, tenso, solo y deprimido.

Para realizar el estudio del bienestar y calidad de vida de las personas mayores de sesenta y cinco años según forma de corresidencia se han definido dos clústeres de países agrupados según la intensidad y frecuencia de este tipo de hogares (Iacovou y Skew, 2011), los valores y actitudes característicos del mapa cultural europeo (Aranda, 2013; Inglehart y Wezel, 2005); los modelos de protección a la tercera edad y la dependencia (Esping Andersen, 1990, p. 199; Pavolini y Ranci, 2008; Rodríguez Cabrero, 2011; Moreno y Serrano, 2009), así como los indicadores de bienestar de este grupo de edad (Litwin, 2010; Steptoe et al., 2014). La combinación de estos factores nos ha permitido agrupar a los países con criterios analíticos probados en estudios anteriores. En principio se obtuvieron tres clústeres, pero en última instancia decidimos presentar en este artículo sólo los dos que tenían mayor interés para nuestro objetivo: 
- Países de baja intensidad de hogares multigeneracionales: Dinamarca, Suecia, Finlandia, Países Bajos e Islandia.

- Países de alta intensidad de hogares multigeneracionales: España, Italia, Grecia y Portugal.

El primer grupo de países aquí presentado se refiere al clúster de los países del norte de Europa. Estos países se caracterizan por una reducida frecuencia de hogares multigeneracionales y por una generosa política social de atención a la tercera edad (Puthenparambil y Kröger, 2016). El segundo clúster estaría formado por los países del sur de Europa (España, Italia, Grecia y Portugal), caracterizados por una moderada frecuencia de hogares multigeneracionales, por la prácticamente ausencia de una política familiar de la dependencia y por la generalización de los denominados valores familistas (Moreno Mínguez, 2013; Naldini, 2003). Partiendo de esta tipificación, el objetivo de este artículo es analizar el bienestar de las personas mayores de 65 años desde la perspectiva del hogar teniendo como eje vertebrador las distintas formas de corresidencia de estas personas en los distintos países. Para tal fin, hemos utilizado como variable de referencia los hogares multigeneracionales en cada grupo de países.

El enfoque metodológico utilizado en este trabajo se ha perfilado desde una doble perspectiva:

- Comparación del bienestar de las personas mayores de 65 años que residen en hogares multigeneracionales entre los clústeres definidos.

- Comparación del bienestar de las personas mayores de 65 años que residen en hogares multigeneracionales frente a los que viven solos o en pareja en cada clúster, y comparación de los resultados obtenidos entre los diferentes clústeres.

La primera fase del análisis se aborda a través del Análisis de Contingencia aplicado a las variables cualitativas y a través del Test de la F de Snedecor si las variables son cuantitativas. La segunda fase del análisis se realiza a través de los Árboles de Decisión, cuya metodología se expone a continuación.

Los Árboles de Decisión presentan múltiples utilidades: segmentación, estratificación, predicción, reducción de datos y clasificación de variables, 


\section{Moreno \& Vicente - Situación Familiar de las Personas Mayores}

identificación de interacción o fusión de categorías y discretización de variables continuas. El método de crecimiento utilizado en este análisis ha sido el CHAID (CHi-square Automatic Interaction Detection). Este procedimiento considera todos los cortes posibles en todas las variables, seleccionando aquél con el menor p-valor asociado al contraste estadístico $\chi^{2}$ de Pearson, si la variable criterio es categórica, o el test de la $F$, si es continua. La búsqueda de la variable y el corte óptimo se lleva a cabo en dos fases:

1)En la fase merge (fusión) se agrupan las categorías de las variables explicativas de cuya unión se obtenga la menor significación estadística (mayor p-valor) asociado al contraste correspondiente, siempre que éste supere un umbral prefijado de antemano, ${ }^{\alpha}$ merge.

2)En la fase split (división) se selecciona la variable de corte, aquella que obtenga la mayor significación estadística (menor p-valor) en el contraste, siempre que éste sea menor a un umbral prefijado, $\alpha_{\text {split }}$.

\section{Discusión de los Resultados}

El análisis de las transformaciones en las tipologías familiares ha sido objetivo de estudio frecuente desde los años 70. Los estudios parecen indicar que las estructuras familiares en Europa se han complejizado, entre otros factores, como consecuencia del envejecimiento de la población y la reducción de la fecundidad.

El primer síntoma de cambio identificado por los historiadores y demógrafos fue la reducción del tamaño medio de los hogares (Burch, 1970; Laslett, 1972). A esto hay que añadir que, dado el envejecimiento de la población y los efectos de la crisis económica, la solidaridad intergeneracional se ha fortalecido en los últimos años (Bengtson, 2001; Hagestad, 2006; Mari-Klose y Escapa, 2015). De hecho, las investigaciones realizadas en Estados Unidos han evidenciado un incremento significativo de los hogares multigeneracionales o extensos en los años de la crisis (Aquilino, 1990; Harrell et al, 2011; Pleau, 2010; Pew Research Center, 2010), aunque la tendencia en términos generales evidencia un claro descenso de este tipo de hogares en todos los países desarrollados (Palloni, 
2001; Pampel, 1992; Ruggles 2007; Tomassini, 2004; United Nations, 2005).

En el caso europeo, los datos disponibles no permiten contrastar que se haya producido un incremento de los hogares multigeneracionales como consecuencia de la crisis económica. Más bien, la tendencia observada lo que muestra es un claro declive de este tipo de hogares desde 1980. Lo que sí que parecen evidenciar los estudios comparados son las diferencias observadas entre el norte y el sur de Europa.

En el caso concreto de España, el modelo de convivencia trigeneracional en el que los nietos y abuelos conviven en el mismo hogar se ha incrementado con la crisis (Laparra y Pérez, 2012).

En los países del norte, la frecuencia de los hogares multigeneracionales es menor que en los países del sur de Europa, debido sobre todo a factores de tipo cultural, histórico e institucional (Rodríguez Laso et al., 2004). Por lo tanto, mientras que la evidencia empírica sugiere un aumento de este tipo de hogares en Estados Unidos (Casper y Bryson, 1998), los datos no parecen evidenciar una tendencia similar en los países europeos, aunque este tipo de estructuras familiares siguen siendo más comunes en el sur que en el norte de Europa (Kozlowski, 2009; Tomassini et al., 2004).

Los datos disponibles muestran una incidencia moderada de los hogares multigeneracionales en los países del sur de Europa. Esta tendencia ha sido paralela al descenso del tamaño medio del hogar como consecuencia de la reducción de la fecundidad, especialmente acentuada en los países del sur de Europa desde mediados de los años 70 . El gráfico 1 evidencia significativas diferencias en la frecuencia de los hogares extensos en los distintos países europeos.

Este tipo de familias apenas tiene relevancia en los países del norte de Europa, representando el $0,2 \%$ en Suecia, 0,6\% en Finlandia, 0,1\% en Dinamarca y el 0,2\% en Países Bajos; siendo más frecuente en los países del sur de Europa, donde estos hogares representan el 2,6\% en Italia, el 4,5\% en España, el 5,5\% en Portugal y el 3,9\% en Grecia. En Europa Central, la frecuencia de este tipo de hogares es más reducida que en los países del sur de Europa pero moderadamente más elevada que en los países nórdicos. 


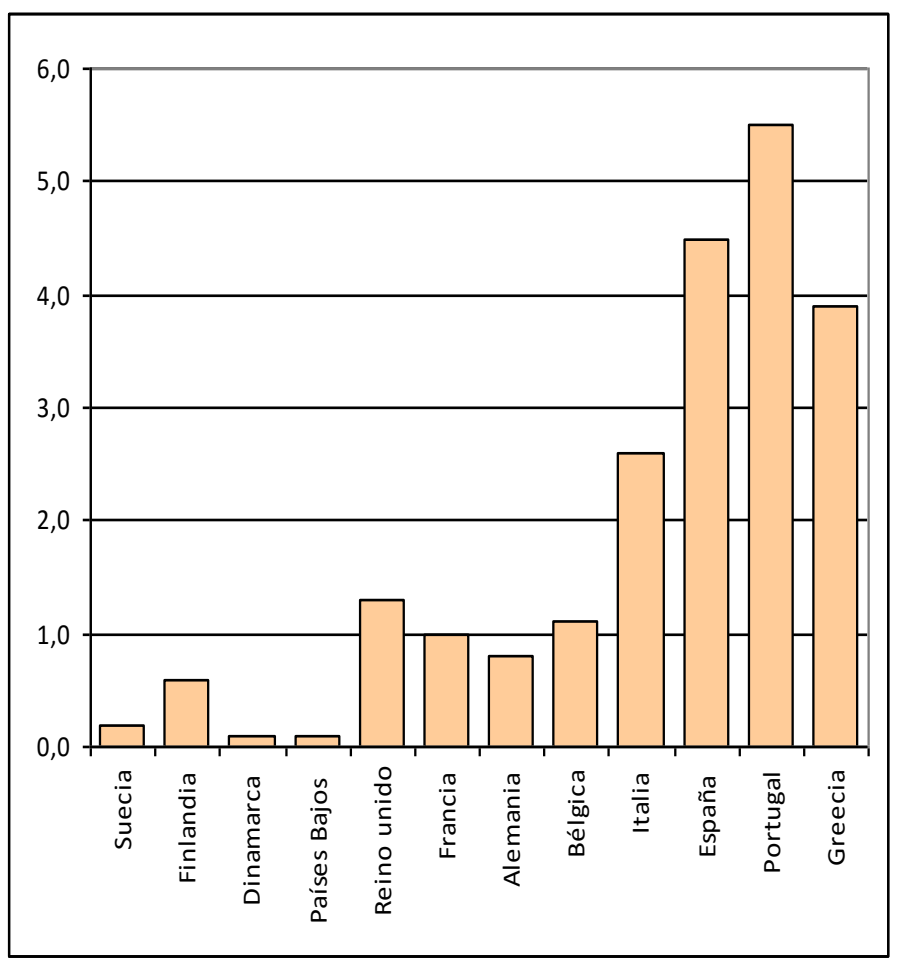

\section{Gráfico 1}

Frecuencia de hogares multigeneracionales en Europa

(\% del total de hogares)

Fuente: Iacovou y Skew (2011)

Históricamente, la mayor frecuencia de este tipo de hogares se ha asociado con unas políticas de servicios sociales de atención a la tercera edad deficitarias, lo que ha redundado en una mayor solidaridad entre generaciones para hacer frente a este déficit de servicios, a diferencia de otros países del norte de Europa, donde es menos frecuente que las familias se ocupen del cuidado de las personas mayores y estos servicios son 
prestados mayoritariamente por las administraciones locales (Daatland, 1997; Haberkern y Szydlik, 2010). Tal y como puede observarse en el gráfico 2, exceptuando la partida de gasto social en pensiones, la ayuda a la dependencia y al cuidado de los ancianos mayores de 65 años en España es una de las más reducidas de Europa. Según estos datos, España tiene el porcentaje más reducido de gasto social en tercera edad y en servicios de cuidado en comparación con los países objeto de análisis. Por el contrario, destacan Dinamarca, Países Bajos y Suecia como aquellos países que más recursos públicos destinan al cuidado y bienestar de la tercera edad.

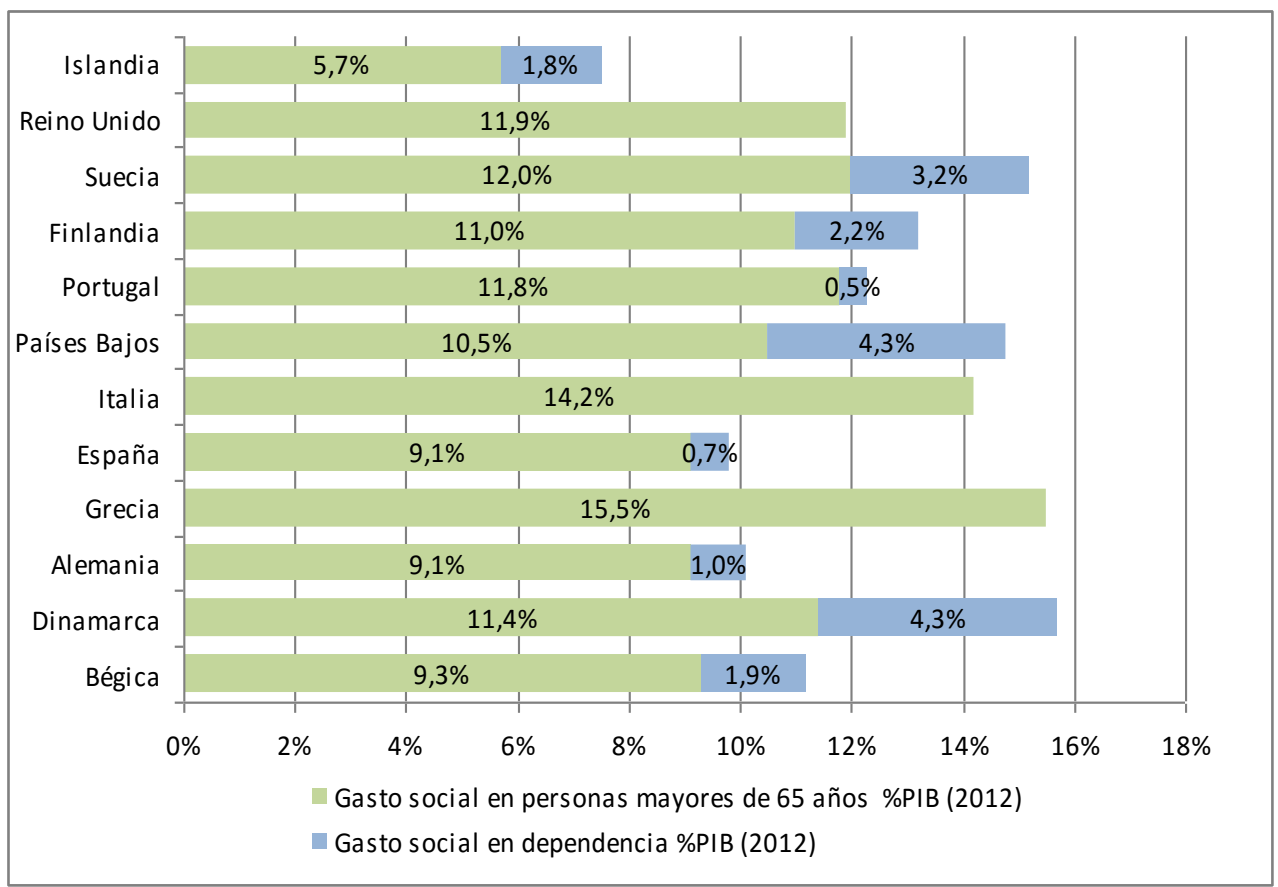

Gráfico 2

Gasto social en tercera edad (\% del PIB), 2012

Fuentes: Eurostat (gasto social), y OCDE Health Care 2015 (gasto en cuidados de larga duración). Elaboración propia. 
Si bien son numerosos los estudios que se han ocupado de la calidad de vida y el estado de salud de las personas mayores de 65 años, son escasos aquéllos que han tenido como fin último medir el bienestar de este grupo utilizando un indicador sintético que integre indicadores de bienestar material, físico, social y emocional (Kaneda, Lee y Pollard, 2011).

En el gráfico 3 se representa el nivel de gasto social de los Estados en relación con el índice de bienestar elaborado por Kaneda, Lee y Pollar (2011) para los países en los que hay datos disponibles. Estos datos no permiten establecer ninguna relación de causalidad, pero sí que describen una situación comparada sobre las políticas sociales y el índice de bienestar.

En el gráfico 3 se observa que aquellos países que más gastan en servicios y atención a la tercera edad, concretamente Dinamarca y Suecia, son los que mayores índices de bienestar tienen entre las personas mayores de 65 años. Por el contrario, España e Italia son los países donde este índice es menor. Destaca el caso de Italia, donde el gasto social es casi similar al de los países del norte de Europa, pero con unos índices reducidos de bienestar. Esto se puede deber a factores propios de la cultura familista que sobrevalora el cuidado y atención de las familias en un momento de cambio en el que las familias, y las mujeres concretamente, ya no pueden ocuparse de la atención de estas personas como en el pasado, debido a su integración en el mercado laboral. De hecho, las dificultades de compatibilización laboral y familiar entre las mujeres son especialmente elevadas en los países del sur de Europa en comparación con los países nórdicos (Moreno Mínguez, 2013).

Para ahondar en las peculiaridades que caracterizan al modelo familiar y al bienestar de este grupo de edad en regímenes de bienestar muy distintos, hemos ideado un modelo analítico en el que se contempla el tipo de hogar en que residen las personas mayores de 65 años y la posible asociación con diferentes dimensiones del bienestar subjetivo. La aportación más destacada de nuestro estudio es que se observan diferencias sustantivas entre el norte y el sur de Europa en la percepción que estas personas tienen del bienestar y la calidad de vida en función del tipo de hogar en el que residen. 


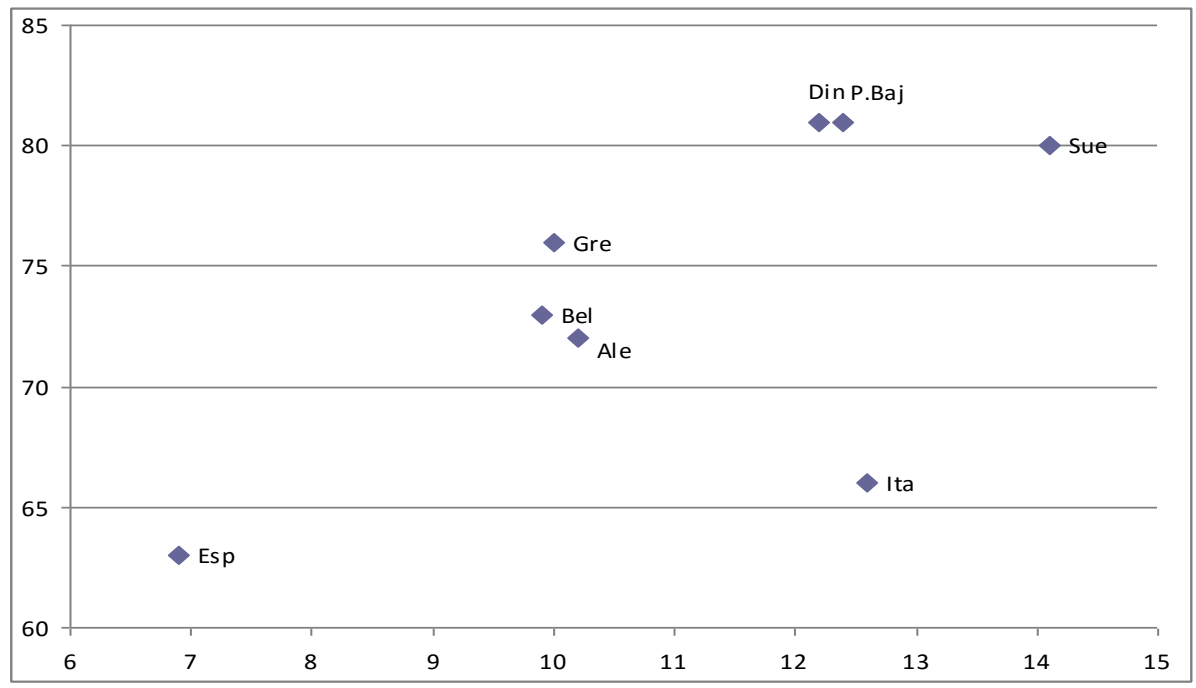

\section{Gráfico 3}

Gasto social en tercera edad (\% PIB) e índice de bienestar, 2006.

Fuentes: Eurostat y OECD Health Data 2008 (gasto social en tercera edad); OCDE Health Care 2015 (gasto en cuidados de larga duración), y Kaneda, Lee y Pollard, 2011 (índice de bienestar). Elaboración propia.

Así, en el clúster 1 correspondiente a los países del norte de Europa (Dinamarca, Suecia, Finlandia, Países Bajos e Islandia), caracterizados por un reducido porcentaje de hogares multigeneracionales y un generoso Estado de bienestar, el bienestar subjetivo se asocia con la valoración positiva de la situación económica del país. Esto podría significar que la crisis económica no ha afectado de forma especial a los servicios públicos de atención a estas personas. En segundo lugar, destaca el hecho de que estas personas en general no se sienten solas, independientemente del tipo de hogar en que residen y de la mayor frecuencia de hogares unipersonales (ver árbol 1). Este hallazgo es coincidente con los resultados de los estudios previos. También destaca el hecho de que se sienten satisfechos con su nivel de vida, lo que se asocia con sentirse tranquilas y relajadas (ver árbol 1). 


\section{Moreno \& Vicente - Situación Familiar de las Personas Mayores}

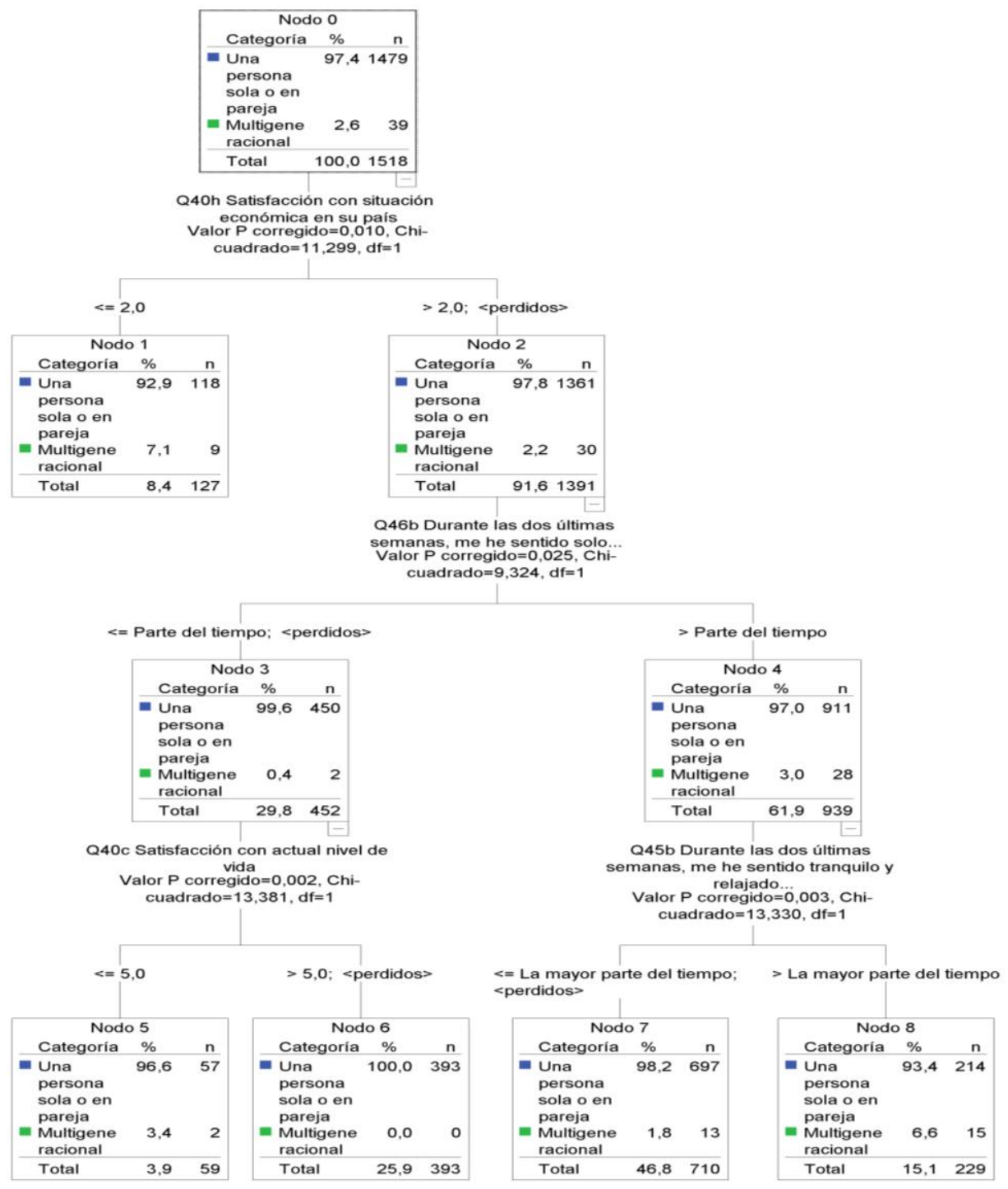

\section{Arbol 1. Países del Norte de Europa}

Fuente: European Quality of Life 2011-2012. Elaboración propia 
Por el contrario, en el clúster 2 referido a los países mediterráneos (España, Italia, Grecia y Portugal), el tipo de hogar en el que residen es un factor clave para explicar el bienestar subjetivo y la calidad de vida de estas personas. Tal y como se puede observar en el árbol 2, la corresidencia en hogares multigeneracionales es la principal variable discriminatoria para no sentirse solo. ${ }^{2}$ Además, estas personas que viven en hogares multigeneracionales se sienten relativamente poco satisfechas con su salud.

Esto se debe en parte a que la familia tiene un papel relevante en sus vidas, y el hecho de vivir solas quizás no ha satisfecho las expectativas de apoyo familiar que tenían y, por tanto, su sentimiento de soledad es mayor. Por otra parte, esto también podría estar afectando a la percepción sobre su estado de salud.

También destaca el hecho de que las personas que se sienten solas tienen una perspectiva más negativa y crítica con la situación económica del país. En conclusión, el factor más diferenciador entre países es la sensación de soledad de estas personas. En los países del sur de Europa, el sentimiento de soledad es menor entre las personas que residen en hogares multigeneracionales, y se muestran además más satisfechas con su estado de salud, al contrario de lo que ocurre con las que viven solas. Estos resultados pueden estar en parte relacionados con la situación de los servicios sociales de la dependencia en estos países y el estado actual de los Estados de bienestar que como consecuencia de los recortes derivados de la crisis económica han reducido las ayudas a la dependencia (Moreno y Mari-Klose, 2013; Rodríguez Cabrero, 2011; Tobío et al., 2010). 
112 Moreno \& Vicente - Situación Familiar de las Personas Mayores

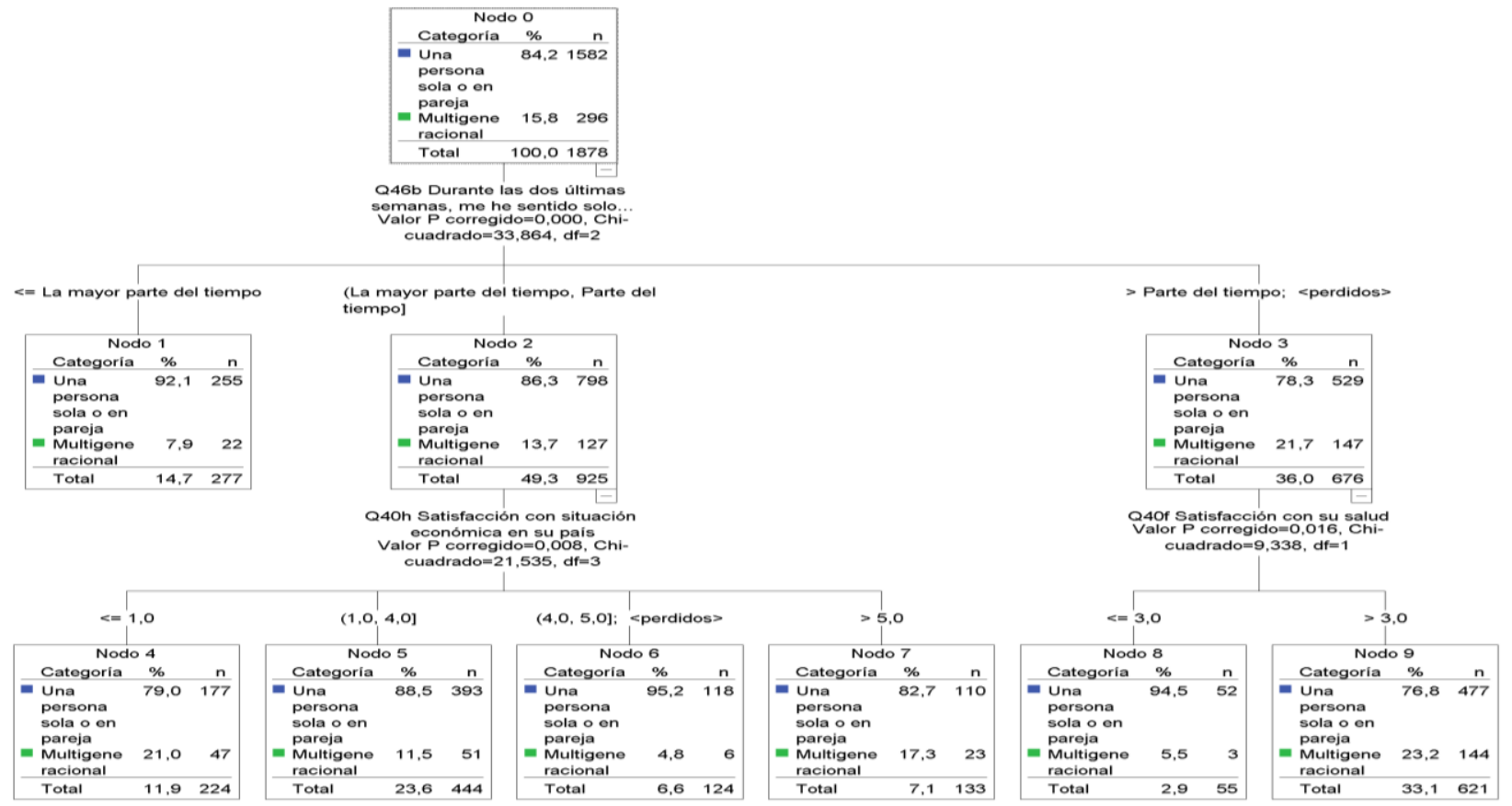

Arbol 2. Países del Sur de Europa

Fuente: European Quality of Life 2011-2012. Elaboración propia 
En definitiva, el análisis comparado sobre el bienestar subjetivo de las personas mayores de 65 años que viven en hogares multigeneracionales revela resultados interesantes para la gestión de las políticas públicas aplicables en cada Estado de bienestar. En primer lugar, destaca que estas personas dedican menos tiempo a los hobbies y al ocio personal en aquellos países con una elevada frecuencia de este tipo de hogares (los países del sur de Europa) (ver tabla 1), lo cual puede redundar en un menor bienestar subjetivo y satisfacción.

Tabla 1

Valoración del tiempo dedicado en pasatiempos propios, intereses (personas mayores de 65 años).

Fuente: European Quality of Life 2011-2012. Elaboración propia

\begin{tabular}{clrrrr}
\hline & & $\begin{array}{c}\text { Menos de } \\
\text { lo que me } \\
\text { gustaría }\end{array}$ & $\begin{array}{c}\text { El que } \\
\text { deseo }\end{array}$ & $\begin{array}{c}\text { Más de lo } \\
\text { que me } \\
\text { gustaría }\end{array}$ & Total \\
\hline \multirow{2}{*}{ Países } & Recuento & 0 & 27 & 13 & 40 \\
nórdicos & Precuencia esperada & 3,0 & 26,3 & 10,7 & 40 \\
& Porcentaje horizontal & $0,0 \%$ & $67,5 \%$ & $32,5 \%$ & $100 \%$ \\
& Residuos corregidos & $-1,9$ & 0,2 & 0,8 & \\
\hline Países de & Recuento & 3 & 67 & 35 & 105 \\
Europa & Frecuencia esperada & 7,8 & 69,0 & 28,2 & 105 \\
central & Porcentaje horizontal & $2,9 \%$ & $63,8 \%$ & $33,3 \%$ & $100 \%$ \\
& Residuos corregidos & $-2,0$ & $-0,5$ & 1,7 & \\
\hline \multirow{2}{*}{ Países del } & Recuento & 29 & 190 & 68 & 287 \\
sur de & Frecuencia esperada & 21,3 & 188,7 & 77,1 & 287 \\
Europa & Porcentaje horizontal & $10,1 \%$ & $66,2 \%$ & $23,7 \%$ & $100 \%$ \\
& Residuos corregidos & 3,0 & 0,3 & $-2,1$ & \\
\hline Total & Recuento & 32 & 284 & 116 & 432 \\
& Porcentaje horizontal & $7,4 \%$ & $65,7 \%$ & $26,9 \%$ & $100 \%$ \\
\hline
\end{tabular}

Chi-cuadrado de Pearson: 11,988 (p-valor=0,017)

Razón de verosimilitudes: 15,462 (p-valor=0,004) 


\section{Moreno \& Vicente - Situación Familiar de las Personas Mayores}

Tal y como puede observarse en la tabla 2, en los países del sur de Europa donde hay una mayor frecuencia de personas mayores de 65 años viviendo en este tipo de hogares, éstas manifiestan una mayor sensación de soledad que las personas de los países del norte de Europa, donde la frecuencia de este tipo de hogares es menor. La sensación de soledad se perfila, pues, como factor clave en la percepción del bienestar en los países mediterráneos, donde los lazos familiares son más fuertes y las familias extensas son más numerosas (Litwin, 2010; Iacovou y Skew, 2011).

Tabla 2

Percepción del sentimiento de soledad. Me he sentido solo en las últimas semanas (personas mayores de 65 años)

Fuente: European Quality of Life 2011-2012. Elaboración propia

\begin{tabular}{|c|c|c|c|c|c|c|c|c|}
\hline & & $\begin{array}{l}\text { Todo el } \\
\text { tiempo }\end{array}$ & $\begin{array}{c}\text { La mayor } \\
\text { parte del } \\
\text { tiempo }\end{array}$ & $\begin{array}{l}\text { Más de la } \\
\text { mitad de } \\
\text { las veces }\end{array}$ & $\begin{array}{l}\text { Menos de } \\
\text { la mitad de } \\
\text { las veces }\end{array}$ & $\begin{array}{l}\text { Parte del } \\
\text { tiempo }\end{array}$ & $\begin{array}{c}\text { En } \\
\text { ningún } \\
\text { momento }\end{array}$ & Total \\
\hline \multirow{4}{*}{$\begin{array}{l}\text { Países } \\
\text { nórdicos }\end{array}$} & Recuento & 0 & 0 & 0 & 1 & 4 & 36 & 41 \\
\hline & Frecuencia esperada & 0,7 & 1,6 & 2,8 & 4,1 & 7,5 & 24,3 & 41 \\
\hline & Porcentaje horizontal & $0,0 \%$ & $0,0 \%$ & $0,0 \%$ & $2,4 \%$ & $9,8 \%$ & $87,8 \%$ & $100 \%$ \\
\hline & Residuos corregidos & $-0,9$ & $-1,3$ & $-1,8$ & $-1,7$ & $-1,5$ & 3,9 & \\
\hline \multirow{4}{*}{$\begin{array}{c}\text { Países de } \\
\text { Europa } \\
\text { central }\end{array}$} & Recuento & 2 & 0 & 2 & 7 & 15 & 83 & 109 \\
\hline & Frecuencia esperada & 1,9 & 4,1 & 7,5 & 10,9 & 19,9 & 64,6 & 109 \\
\hline & Porcentaje horizontal & $1,8 \%$ & $0,0 \%$ & $1,8 \%$ & $6,4 \%$ & $13,8 \%$ & $76,1 \%$ & $100 \%$ \\
\hline & Residuos corregidos & 0,0 & $-2,4$ & $-2,4$ & $-1,4$ & $-1,4$ & 4,1 & \\
\hline \multirow{4}{*}{$\begin{array}{c}\text { Países del } \\
\text { sur de } \\
\text { Europa }\end{array}$} & Recuento & 6 & 17 & 29 & 37 & 63 & 147 & 299 \\
\hline & Frecuencia esperada & 5,3 & 11,3 & 20,6 & 30,0 & 54,6 & 177,1 & 299 \\
\hline & Porcentaje horizontal & $2,0 \%$ & $5,7 \%$ & $9,7 \%$ & $12,4 \%$ & $21,1 \%$ & $49,2 \%$ & $100 \%$ \\
\hline & Residuos corregidos & 0,5 & 3,0 & 3,3 & 2,3 & 2,2 & $-6,1$ & \\
\hline \multirow{2}{*}{ Total } & Recuento & 8 & 17 & 31 & 45 & 82 & 266 & 449 \\
\hline & Porcentaje horizontal & $1,8 \%$ & $3,8 \%$ & $6,9 \%$ & $10,0 \%$ & $18,3 \%$ & $59,2 \%$ & $100 \%$ \\
\hline
\end{tabular}

Chi-cuadrado de Pearson: 45,184 (p-valor $=0,000)$

Razón de verosimilitudes: 56,010, (p-valor=0,000) 
Esto también parece repercutir en una menor satisfacción con su estado de salud. De hecho, la percepción del estado de salud es relativamente inversa a la intensidad de los hogares multigeneracionales (ver tabla 3). Por otro lado, en los países con una menor proporción de hogares multigeneracionales, la percepción subjetiva del estado de salud es más positiva. En consonancia con este hallazgo se podría explicar el hecho de que las personas de más edad que viven en los países del norte de Europa se sienten con mejor estado de ánimo (ver tabla 4).

Tabla 3

Percepción sobre el estado de salud (personas mayores de 65 años)

Fuente: European Quality of Life 2011-2012. Elaboración propia

\begin{tabular}{clrrrrrr}
\hline & & $\begin{array}{c}\text { Muy } \\
\text { bueno }\end{array}$ & Bueno & Aceptable & Malo & $\begin{array}{c}\text { Muy } \\
\text { malo }\end{array}$ & Total \\
\hline \multirow{4}{*}{$\begin{array}{c}\text { Países } \\
\text { nórdicos }\end{array}$} & 9 & 13 & 12 & 7 & 0 & 41 \\
& Recuento & 5,2 & 14,1 & 16,1 & 4,4 & 1,3 & 41 \\
& Porcentaje horizontal & $22,0 \%$ & $31,7 \%$ & $29,3 \%$ & $17,1 \%$ & $0,0 \%$ & $100 \%$ \\
& Residuos corregidos & 1,9 & $-0,4$ & $-1,4$ & 1,4 & $-1,2$ & \\
\hline \multirow{4}{*}{$\begin{array}{c}\text { Países de } \\
\text { Europa } \\
\text { central }\end{array}$} & Recuento & 25 & 35 & 32 & 10 & 8 & 110 \\
& Precuencia esperada & 13,9 & 37,8 & 43,2 & 11,7 & 3,4 & 110 \\
& Residuos corregidos & 3,7 & $-0,6$ & $-2,5$ & $-0,6$ & 2,9 & \\
\hline \multirow{2}{*}{$\begin{array}{c}\text { Países } \\
\text { del sur de herizontal }\end{array}$} & $22,7 \%$ & $31,8 \%$ & $29,1 \%$ & $9,1 \%$ & $7,3 \%$ & $100 \%$ \\
Europa & Recuento & 23 & 107 & 133 & 31 & 6 & 300 \\
& Precuencia esperada & 37,9 & 103,1 & 117,7 & 31,9 & 9,3 & 300 \\
& Residuos corregidos & $-4,5$ & 0,8 & 3,1 & $-0,3$ & $-1,9$ & \\
\hline \multirow{2}{*}{ Total } & Recuento & 57 & 155 & 177 & 48 & 14 & 451 \\
& Porcentaje horizontal & $12,6 \%$ & $34,4 \%$ & $39,2 \%$ & $10,6 \%$ & $3,1 \%$ & $100 \%$ \\
\hline
\end{tabular}

Chi-cuadrado de Pearson: 34,366 (p-valor $=0,000$ )

Razón de verosimilitudes: 32,926 (p-valor=0,000) 
Tabla 4

Percepción sobre el estado de ánimo (personas mayores de 65 años)

Fuente: European Quality of Life 2011-2012. Elaboración propia

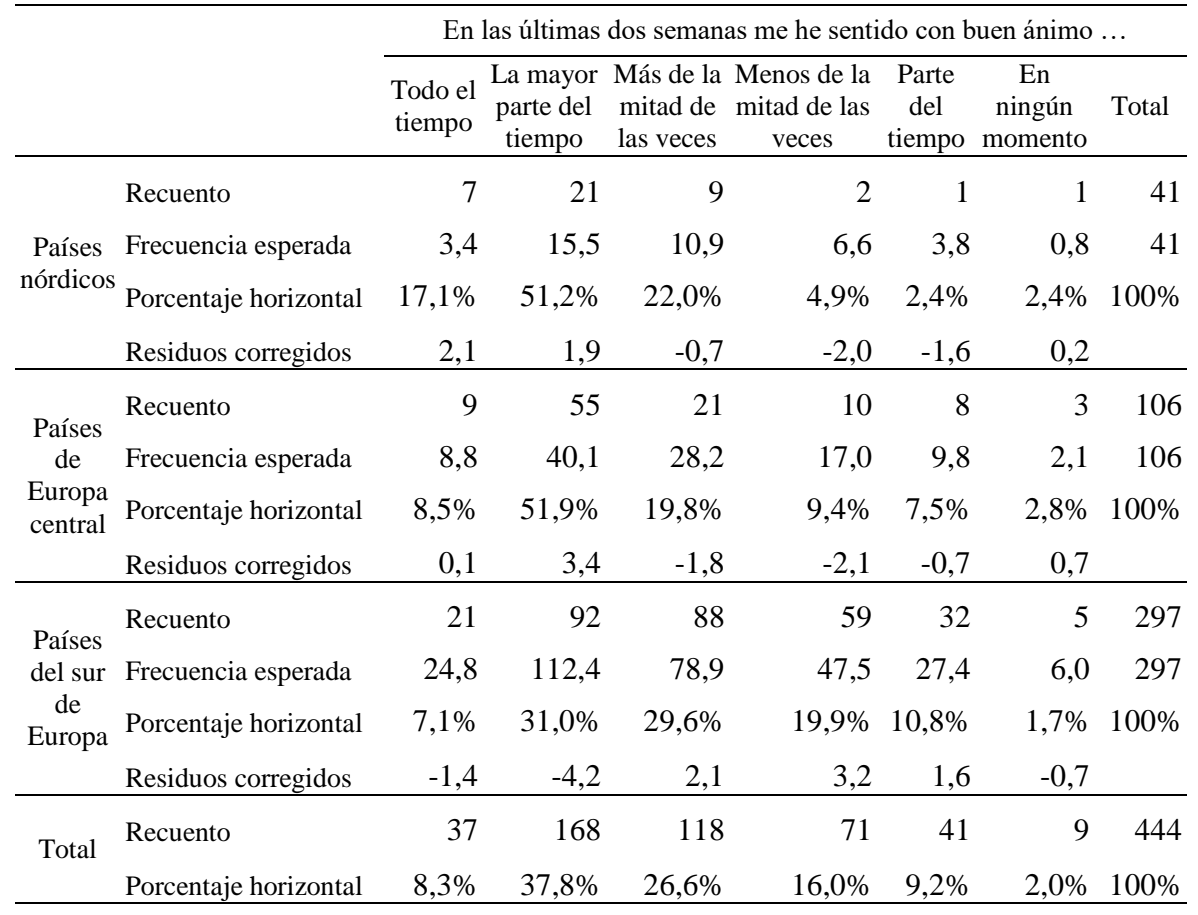

Chi-cuadrado de Pearson: 31,180 (p-valor $=0,001$ )

Razón de verosimilitudes: 32,266 (p-valor=0,000)

Finalmente, se observa que la satisfacción con las condiciones económicas personales y su percepción de la felicidad es menor en los países del sur de Europa, donde los hogares multigerancionales son más frecuentes y donde los efectos de la crisis económica han sido muy severos (ver tabla 5). Esto puede estar relacionado con el hecho de que la mayor parte de los nuevos hogares multigeneracionales creados en los países del sur de Europa durante la crisis han sido resultado de una reagrupación familiar motivada por la pérdida de empleo y/o poder adquisitivo de alguno/s de sus miembros 
asociada al actual contexto de crisis económica, aspecto también importante en los países mediterráneos, donde la crisis económica ha azotado con mayor virulencia y parece haber reducido las redes de solidaridad familiar (Martínez Virto, 2014). Los resultados relativos a este clúster constatan que las personas mayores de 65 años residentes en los países del sur de Europa se declaran más insatisfechas con su nivel de vida y menos tranquilas y relajadas que las personas de la misma edad residentes en los países nórdicos (ver árbol 2).

\section{Tabla 5}

Satisfacción con diferentes dimensiones (escala de 1 a 10). (personas mayores de 65 años)

Fuente: European Quality of Life 2011-2012. Elaboración propia

\begin{tabular}{|c|c|c|c|c|c|c|c|c|c|c|c|c|}
\hline & \multicolumn{3}{|c|}{ Países nórdicos } & \multicolumn{3}{|c|}{$\begin{array}{c}\text { Países de Europa } \\
\text { central }\end{array}$} & \multicolumn{3}{|c|}{$\begin{array}{l}\text { Países del sur de } \\
\text { Europa }\end{array}$} & \multicolumn{3}{|c|}{ Total } \\
\hline & Media & $\mathrm{N}$ & Desv & Media & $\mathrm{N}$ & $\begin{array}{l}\text { Desv } \\
\text { típica }\end{array}$ & Media & $\mathrm{N}$ & $\begin{array}{l}\text { Desv } \\
\text { típica }\end{array}$ & Media & $\mathrm{N}$ & $\begin{array}{l}\text { Desv } \\
\text { típica }\end{array}$ \\
\hline $\begin{array}{l}\text { Satisfacción con } \\
\text { actual nivel de } \\
\text { vida }\end{array}$ & 7,94 & 41 & 2,25 & 7,63 & 109 & 1,95 & 6,81 & 300 & 2,09 & 7,11 & 450 & 2,11 \\
\hline $\begin{array}{l}\text { Satisfacción con } \\
\text { su alojamiento }\end{array}$ & 8 & 41 & 1,8 & 8,28 & 109 & 1,59 & 7,78 & 300 & 1,98 & 7,96 & 450 & 1,90 \\
\hline $\begin{array}{l}\text { Satisfacción con } \\
\text { sit. económica } \\
\text { en su país }\end{array}$ & 4,79 & 41 & 2,64 & 4,56 & 104 & 2,19 & 3,03 & 298 & 2,06 & 3,55 & 443 & 2,28 \\
\hline $\begin{array}{l}\text { Felicidad en } \\
\text { general }\end{array}$ & 8,15 & 41 & 1,38 & 7,79 & 108 & 1,84 & 7,15 & 300 & 1,75 & 7,39 & 450 & 1,78 \\
\hline
\end{tabular}

En definitiva, el análisis realizado subraya diferencias sustanciales en la percepción del bienestar y la calidad de vida de las personas mayores de 65 años en función del país de residencia y el tipo de hogar en el que residen. En los países del norte de Europa la percepción de bienestar y calidad de vida es mayor, independientemente del tipo de hogar en que se resida, mientras que, en los países del sur de Europa, vivir en hogares 


\section{Moreno \& Vicente - Situación Familiar de las Personas Mayores}

multigeneracionales se asocia con un mayor sentimiento de soledad, y menor bienestar y calidad de vida.

Estos resultados cuestionarían en parte la idea generalizada de que en los países del sur de Europa la solidaridad familiar entre generaciones favorece el bienestar de las personas mayores, ya que los resultados obtenidos en los países del norte de Europa apuntan a que podrían ser las políticas públicas destinadas a este colectivo las que favorecen un mayor bienestar y calidad de vida.

\section{Conclusiones}

La literatura existente se ha basado fundamentalmente en analizar la relación entre los diferentes indicadores de salud, la calidad de vida y el bienestar objetivo de la tercera edad. La principal aportación de este estudio es la introducción de la variable familiar para explicar la diferente percepción de bienestar y calidad de vida en los distintos clústeres de países analizados.

Los resultados obtenidos destacan que el sentimiento de soledad se asocia con una peor percepción sobre el estado de salud, siendo esta sensación mayor entre las personas de los países del sur de Europa, donde hay una mayor frecuencia de hogares multigeneracionales.

Esta percepción de menor bienestar y calidad de vida es más elevada en los países del sur de Europa que en los del norte de Europa, donde el sentimiento de soledad es menor y donde el bienestar se asocia con factores tales como el nivel económico de vida.

Estas conclusiones generales apuntan indirectamente al papel que ha desempeñado el Estado de bienestar y la familia en el bienestar subjetivo y calidad de vida de estas personas en los diferentes países europeos. Así:

- En los países mediterráneos, donde el Estado de bienestar y las políticas sociales de atención a este grupo de edad han sido limitadas, la familia se ha convertido en el principal soporte asistencial y emocional de estas personas, aunque esto, en términos comparados, no se traduzca en un mayor sentimiento de bienestar. En un contexto en el que las familias ya no pueden hacerse cargo de estos familiares, bien porque la mujer trabaja o porque no tienen recursos terapéuticos 
o económicos suficientes, el bienestar y calidad de vida de estas personas se pueden estar viendo afectados gravemente.

- Por el contrario, en los países del norte y del centro Europa, donde el Estado de bienestar ha sido más generoso y favorecedor de la institucionalización de la dependencia, el bienestar no se remite únicamente a la solidaridad familiar, sino al nivel de vida que garantizan las políticas y los servicios públicos, tal y como se ha evidenciado en este estudio.

Por lo tanto, estos resultados sugieren que los gobiernos de los Estados del bienestar del sur de Europa deben de estar atentos a los cambios familiares y a las políticas sociales destinadas a la tercera edad para favorecer la calidad de vida y el bienestar de estas personas en sociedades cada vez más envejecidas. No debemos olvidar que este colectivo representa una parte importante en las pirámides de población como consecuencia del progresivo envejecimiento de la población.

En definitiva, los Estados del bienestar en el Sur de Europa, si quieren apostar por el bienestar y la calidad de vida de las personas mayores de 65 años, deberían revisar las agendas de las políticas sociales destinadas a este colectivo.

\section{Notas}

${ }^{1}$ Para más información sobre las variables que contiene el cuestionario ver:

http://www.eurofound.europa.eu/surveys/european-quality-of-life-surveys-eqls/eqls2012/eqls-2012-questionnaire.

${ }^{2}$ Precisamente las personas mayores de 65 años que viven solos o en pareja se sienten solas en mayor medida que las personas que residen en este tipo de hogar en los países nórdicos.

\section{Referencias}

Aranda, L. (2013). Doubling up: a gift or a same? Multigenerational household and parental depression of older Europeans. Venice: Department of Economics. University of Venice. 
Alburquerque, P. C. (2011). Grandparents in multigenerational households:

The case of Portugal. European Journal of Ageing, 8, 189-198. Doi:

10.1007/s10433-011-0196-2

Aquilino, W. S. (1990). The likelihood of parent-adult child coresidence:

Effects of family structure and parental characteristics. Journal of

Marriage and the Family, 52, 405- 419.

http://www.jstor.org/stable/353035

Bengston, V. L. (2001). Beyond the nuclear family: the increasing

importance of multigenerational relationships in American society.

Journal of Marriage and the Family, 63, 1-16. Doi: 10.1111/j.1741-

3737.2001.00001.x

Bettio, F., Simonazzi, A., \& Villa, P. (2006). Change in care regimes and female migration: The 'care drain' in the Mediterranean. Journal of European Social Policy, 16, 271-285. Doi: 10.1177/0958928706065598

Bowling, A. (1997). Measuring health: A review of quality of life measurement scales. Buckingham: Open University Press.

Burch, T. K. (1970). Some demographic determinants of average household size: An analytic approach. Demography, 7, 61-70. Doi:

$10.2307 / 2060023$

Calzada, I. (2013). The Myth of Mediterranean Familism: family values, family structure and public preferences for state intervention in care. European Societies, 15(4), 514-534. Doi:

10.1080/14616696.2013.836402

Carrascosa, L. (2011). Articulación entre las relaciones familiares y sociales y la discapacidad en Europa. Madrid: Fundación Alternativas.

Carrascosa, L. \& Sancho, M. T. (2013). Formas de convivencia, relaciones entre personas y la experiencia de envejecer. En: Díaz Martín, Rosa, Las personas mayores en España: Informe 2010. Madrid: Ministerio de Trabajo y Asuntos Sociales, IMSERSO.

Casper, L. M. \& Bryson, K. R. (1998). Co-resident grandparents and their grandchildren: Grandparent maintained families. Working paper, 26. Washington, D.C.: Population Division, U.S. Bureau of the Census. CDC (2013). Quality of life. Centers for disease control and prevention. Georgia: Atlanta.

Connell, J., Brazier, J., O'Cathain, A., Lloyd-Jones, M., \& Paisley, S. (2012). Quality of life of people with mental health problems: a synthesis 
of qualitative research. Health and Quality of Life Outcomes, 10(138), 116. Doi: 10.1186/1477-7525-10-138

Daatland, S. O. \& Herlofson, K. (2003). 'Lost solidarity' or 'changed solidarity': a comparative European view of normative family solidarity. Ageing \& Society, 23, 537-560. Doi: http://dx.doi.org/10.1017/S0144686X03001272

Daatland, S. O. (1997). Welfare policies for older people in transition? Emerging trends and comparative perspectives. International Journal of Social Welfare, 6(3), 153-161. Doi: 10.1111/j.1468-2397.1997.tb00184.x Deaton, A. (2008). Income, health, and well-being around the world: evidence from the Gallup World Poll. J Econ Perspect, 22, 53-72. Doi: http://dx.doi.org/10.1257/089533008785492315

Diener, E. (2009). The Evolving Concept of Subjective Well-Being: The Multifaceted Nature of Happiness, Assessing Well-Being. Social Indicators Research Series, 39, 67-100. Doi: 10.1007/978-90-481-23544

Dolan, P., Peasgood, T., \& White, M. (2008). Do we really know what makes us happy? A review of the economic literature on the factors associated with subjective well-being. Journal of Economic Psychology, 29, 94-122. Doi: 10.1016/j.joep.2007.09.001

Erlinghagen, M., \& Hank, K. (2006). The participation of older Europeans in volunteer work. Ageing \& Society, 26, 567-584. Doi:

http://dx.doi.org/10.1017/S0144686X06004818

Esping-Andersen, G. (1990). The Three Worlds of Welfare Capitalism. Cambridge: Policy Press.

Esping-Andersen, G. (1999). Social foundations of postindustrial economies. Oxford: University Press Oxford

Eurofound (2014). Quality of Life in Europe: Families in the economic crisis. Luxemburg: European Union.

Ferrera, M. (1996). The 'Southern Model' of Welfare in Social Europe. Journal of European Social Policy, 6(1), 17-37.

Fernández-Ballesteros, R. (2002). Social support and quality of life among older people in Spain. Journal of Social Issues, 58, 645-661. Doi: $10.1111 / 1540-4560.00282$ 
Fokema, T. \& Liefbroer, A. (2008). Trends in living arrangements in Europe: Convergence or divergence? Demographic Research, 19, 13511418. Doi: 10.4054/DemRes.2008.19.36

Green, B. H., Copeland, J. R., Dewey, M. E., Shamra, V., Saunders, P. A., Davidson, I. A., Sullivan, C., \& McWilliam, C. (1992). Risk factors for depression in elderly people: A prospective study. Acta Psychiatr Scand, 86(3), 213-7. Doi: 10.1111/j.1600-0447.1992.tb03254.x

Haberkern, K., \& Szydlik, M. (,2010). State care provision, societal opinion and children's care of older parents in 11 European countries. Ageing \& Society, 30, 299-323. Doi:

http://dx.doi.org/10.1017/S0144686X09990316

Hafiz, T. A., Khan, \& Robert Raeside (2014). Between Country Variations in Self-Rated-Health and Associations with the Quality of Life of Older People: Evidence from the Global Ageing Survey. Applied Research Quality of Life, 9, 923-949. Doi: 10.1007/s11482-013-9278-y

Hagestad, D. (2006). Transfers between grandparents and grandchildren: Theimportance of taking a three-generation persepctive. Zeitschrift Fur Familienforschung, 18, 315-332.

Hammel, E.A. \& Laslett, P. (1974). Comparing household structure over time and between cultures. Comparative Studies in Society and History 16(1), 73-109. Doi: http://dx.doi.org/10.1017/S0010417500007362 Hank, K. \& Buber, I. (2009). Grandparents Caring for Their Grandchildren Findings From the 2004 Survey of Health, Ageing, and Retirement in Europe. Journal of Family Issues, 30, 53-73. Doi:

10.1177/0192513X08322627

Hantrais, L., Philipov, D. \& Billari, F.C. (2006). Policy implications of changing family formation. Population Studies 49. Strasbourg: Council of Europe Publishing.

Harrell, R., Kassner, E. \& Figueiredo, C. (2011). Multigenerational households are increasing. Washington, D.C.: AARP Public Policy Institute.

Iacovou, M. \& Skew, A. (2011). Household composition across the new Europe: Where do the new Member States fit in? Demographic Research, 25(1), 465-490. Doi: http://dx.doi.org/10.4054/DemRes.2011.25.14 Inglehart, R. (ed.) (2003). Human values and social change. Leiden-Boston: Brill. 
Inglehart, T. \& Welzel, Ch. (2005). Modernization, Cultural Change, and Democracy: The Human Development Sequence. Cambridge: Cambridge University Press.

Kahn, Joan R., Frances Goldscheider, \& Garcia-Manglano, J. (2013).

Growing Parental Economic Power in Parent-Adult Child Households:

Coresidence and Financial Dependency in the United States, 1960-2010.

Demography, 50, 449-75.

http://link.springer.com/article/10.1007/s13524-013-0196-2

Kalmijn, M., \& Saraceno, C. (2008). A comparative perspective on intergenerational support-Responsiveness to parental needs in individualistic and familialistic countries. European Societies, 10, 479508. Doi: 10.1080/14616690701744364

Kaneda, T., Lee, M. \& Pollard, K. (2011). SCL/PRB Index of Well-Being in Older Populations, Final Report, Global Aging and Monitoring Project, The Stanford Center on Longevity and the Population Reference Bureau. Kapteyn, A., Lee, J., Tassot, C., Vonkova, H., \& Zamarro, G. (2015).

Dimensions of Subjective Well-Being. Social Indicators Research, 123(3), 625-660. Doi: 10.1007/s11205-014-0753-0

Kass, Gordon V. (1980). An Exploratory Technique for Investigating Large Quantities of Categorical Data. Applied Statistics, 29(2), 119-127. Doi: 10.2307/2986296

Keilman, N. (1987). Recent trends in family and household composition in Europe. European Journal Population, 3, 297-325. Doi:

10.1007/BF01796903

Kimm, J., Lii T., Nai Peng T., \& Sor Tho, N. (2014). Family Support and Loneliness among Older Persons in Multiethnic Malaysia. The Scientific World Journal, 2014, 1-11. Doi: http://dx.doi.org/10.1155/2014/654382 Koslowski, S. A. (2009). Grandparents and the care of their grandchildren. En: Stillwell, J. (ed.), Fertility, living arrangements, care and mobility. London: Springer.

Land, Kenneth C., Michalos, Alex C., \& Sirgy, Joseph (eds) (2012). Handbook of Social Indicators and Quality of Life Research. Springer. Laparra, M. \& Pérez, B. (2012). Crisis y fractura social en Europa: Causas y efectos en España, colección de estudios sociales 35. Barcelona: Obra social La Caixa. 
Laslett, P. (1972). Mean household size in England since the sixteenth century. En: Laslett, P. \& Wall, R. (eds). Household and Family in past time (121-158). London: Cambridge University Press.

Leibfried, S. (1992). Towards a European Welfare State? On integrating Poverty Regimes into the European Community. En Ferge, Z. \& J. Kolberg (eds), Social Policy in a Changing Europe (245-279). Boulder, CO: Westview.

Leeson, W. (2013). Levels of welfarism and intergenerational transfers within the family: evidence from the Global Ageing Survey (GLAS) (with Khan, H.). En: McDaniel, S. A. \& Zimmer, Z. (eds), Global Ageing in the Twenty-First Century - Challenges, Opportunities and Implications (291-308). Burlington USA: Ashgate.

Litwin, H. (2010). Social networks and well-being: a comparison of older people in Mediterranean and non-Mediterranean countries. Journals of Gerontology B: Psychological Sciences and Social Sciences, 65(5), 599608. Doi: 10.1093/geronb/gbp104

López Garcia, E., Banegas, J., Graciani Perez, A., Herruzo, R. \& RodriguezArtalejo, F. (2005). Social network and health-related quality of life in older adults: a population-based study in Spain. Quality of Life Research, 14(2), 511-520. Doi: doi:10.1007/s11136-004-5329-z

Marí-Klose, M. \& Escapa Solanas, S. (2015). Solidaridad intergeneracional en época de crisis: ¿mito o realidad? Panorama Social, 22, 61-78.

Martínez Virto, L. (2014). Crisis en familia. Síntomas de agotamiento de la solidaridad familiar, VII Informe sobre exclusión y desarrollo en España 2014. Madrid: Fundación Foessa, Cáritas.

Moreno, L. \& Serrano, A. (2009). The European Social Model and social policies: a formative institutional evaluation. Gestión y Análisis de Políticas Públicas, 2, 11-32.

Moreno, L. y Marí-Klose, P. (2013). Las transformaciones del Estado del Bienestar Mediterráneo: Trayectorias y retos de un régimen en transición. En: Del Pino, E. y Rubio, M. J. (eds), Los Estados de Bienestar en la encrucijada. Políticas sociales en perspectiva comparada (pp. 126-146). Madrid: Tecnos.

Moreno Mínguez, A. (ed.) (2013). Family wellbeing. European Perspectives. London: Springer. 
Moreno Mínguez, A., Fernández, L. \& Carrasco, A. (2014). Family Policy Indicators and Well-Being in Europe from an Evolutionary Perspective. Journal of Applied Research in Quality of Life, 11(2), 343-367. Doi:

10.1007/s11482-014-9326-2

Naldini, M. (2013). The family in the Mediterranean Welfare States. London: Frank Class.

OECD (2011). Doing better for families. París: OECD.

www.oecd.org/social/family/doingbetter.

OECD (2010a). Improving health and social cohesion through education. Paris: OECD.

OECD (2010b). Society at a glance. Paris: OECD.

Ogg, J. (2005). Social exclusion and insecurity among older Europeans. The influence of welfare regimes. Ageing and Society, 25, 69-90.

Otero, A., Zunzunegui, M., Rodríguez-Laso, A., Aguilar, M., \& Lázaro, P.

(2004). Volumen y tendencias de la dependencia asociada al envejecimiento en la población española. Rev Esp Salud Pública, 78, 201-213.

Palloni, A. (2001). Living Arrangements of Older Persons: Critical Issues and Policy Responses. Popul. Bulletin of the United Nations (54-110). New York: United Nations.

Pampel, F. C. (1992). Trends in Living Alone among the Elderly in Europe. Elderly Migration and Population Redistribution. A comparative study. London: Belhaven.

Pavolini, E. \& Ranci, C. (2008). Restructuring the Welfare State: reforms in long term care in Western European countries. Journal of European Social Policy, 18(3), 246-259. Doi: 10.1177/0958928708091058 Pew Research Centre (2010). The Return of the Multi-Generational Family Household. Washington: Research Center's Social \& Demographic Trends Project.

Pleau, R. L. (2010). Trends and correlates of multigenerational coresidence, 1989-2009. PI Workshop. University of California Davis, Department of Sociology.

Puthenparambil, J. \& Kröger, T. (2016). Using Private Social Care Services in Finland: Free or Forced Choices for Older People? Journal of Social Service Research, 42(2), 167-179. Doi: 10.1080/01488376.2015.1137534 
Reher, D. S. (1998). Family ties in Western Europe: Persistent contrasts.

Population and Development Review, 24(2), 203-234. Doi:

10.2307/2807972

Rodríguez Cabrero, G. (2011). Políticas sociales de atención a la dependencia en los Regímenes de Bienestar de la Unión Europea.

Cuadernos de Relaciones Laborales, 29(1), 13-42.

Rodríguez Laso, A., Zunzunegui, M. \& Otero, A. (2004). CLESA Project no. QLK6-CT-2000-00664. Deliverable 5: Report on Cross-national Comparison of the Impact of Social Networks on the Health Status of the Elderly (Work Package 4), Vth Framework Programme for Research. Bruselas: Unión Europea.

Ruggles, S. (2007). The Decline of Intergenerational Coresidence in the United States, 1850 to 2000. American Sociological Review, 72, 964-989. Doi: 10.1177/000312240707200606

Saraceno, C. (2008). Patterns of family living in the enlarged EU. En: Alber, J., Fahey, T., \& Saraceno, C. (eds), Handbook of Quality of Life in the Enlarged European Union (47-72). London and New York: Routledge. Singh, A. \& Misra, N. (2009). Loneliness, depression and sociability in old age. Ind Psychiatry, 18(1), 51-55.

Smith, J., Borchelt, M., Maier, H., \& Jopp, D. (2002). Health and well-being in the young old and oldest old. Journal of Social Issues, 58(4), 715-732. Doi: 10.1111/1540-4560.00286

Springer, S. (2010). Living conditions and life satisfaction of older Europeans living alone: a gender and cross-country analysis. Ageing and Society, 30, 1153-1175. Doi:

http://dx.doi.org/10.1017/S0144686X10000231

Steptoe, A., Deaton, A., \& Stone, A. (2014). Subjective wellbeing, health, and ageing. The Lancet, 385(9968), 640-648. Doi: 10.1016/S01406736(13)61489-0

Stillwell, J. (ed.) (2009). Fertility, living arrangements, care and mobility. London: Springer

Stone, A., Joseph E., Schwartz, J. E., Brodericka, \& Angus Deaton (2010).

A snapshot of the age distribution of psychological well-being in the United States. Proceedings of the National Academy of Sciences of the United States of America, 107(22), 9985-9990. 
Tobío Soler, C., Agulló Tomás, M., Gómez, M. \& Martín Palomo, M. (2010). El cuidado de las personas: un reto para el siglo XXI. Barcelona: Obra Social La Caixa.

Tomassini, C., Glaser, K., Wolf, D. A., Van Groenou, M. I. B. \& Grundy, E. (2004). Living arrangements among older people: an overview of trends in Europe and USA. Further release of 2001 Census data, 1329, 24-34. Ulloa, B., Møller, V, \& Sousa-Poza, A. (2013). How does subjective wellbeing evolve with age? A literature review. IZA Discussion Paper No. 7328.

Van Tilburg, T., De JongGierveld, J., Lecchini, L. \& Marsiglia, D. (1998). Social integration and loneliness: a comparative study among older adults in the Netherlands and Tuscany, Italy. Journal of Social and Personal Relationships, 15(6), 740-754. Doi: 10.1177/0265407598156002

Viazzo, P. (2003). What's so special about the Mediterranean? Thirty years of research on household and family in Italy. Continuity and Change, 18, 111-137. Doi: http://dx.doi.org/10.1017/S0268416003004442

Wikman, A., Wardle, J. \& Steptoe, A. (2011). Quality of Life and Affective Well-Being in Middle-Aged and Older People with Chronic Medical Illnesses: A Cross-Sectional Population Based Study, PLoS ONE, 6(4). Doi: http://dx.doi.org/10.1371/journal.pone.0018952

United Nations (2007). Principles and recommendations for population and housing censuses. Revision 2. New York: United Nations.

Yang, X., Hao, Y., George, S. M., \& Wang, L. (2012). Factors associated with health-related quality of life among Chinese caregivers of the older adults living in community: a cross-sectional study. Health and Quality of Life Outcomes, 10(143), 1-12. Doi: 10.1186/1477-7525-10-143 Zueras, P. \& Pau Miret, P. (2013). Mayores que viven solos. Una panorámica a partir de los censos de 1991 y 2001. Revista Española de Investigaciones Sociológicas, 144, 139-152. Doi: http://dx.doi.org/10.5477/cis/reis.144.139

Almudena Moreno Mínguez, Universidad de Valladolid (España) Juan Antonio Vicente Virseda, Universidad Nacional de Educación a Distancia (España)

Contact Address: almudena@soc.uva.es 